\title{
Morphometric Analysis of Lumbar Arteries and Relationship with Intervertebral Discs: A study of Surgical Anatomy on Human Fresh Cadavers
}

\author{
Halil CAN1 ${ }^{*}$, Furkan DIREN², Baris PEKER ${ }^{3}$, Cengiz GOMLEKSIZ ${ }^{4}$, Dogan Gucluhan GUCLU5 ${ }^{5}$, Ceyhun KUCUK ${ }^{6}$, \\ Erdinc CIVELEK ${ }^{2}$, Aydin AYDOSELI ${ }^{3}$, Altay SENCER ${ }^{3}$ \\ 'Biruni University, School of Medicine, Department of Neurosurgery, Istanbul, Turkey \\ ${ }^{2}$ Istanbul Gaziosmanpasa Taksim Training and Research Hospital, Department of Neurosurgery, Istanbul, Turkey \\ ${ }^{3}$ Istanbul University, Istanbul School of Medicine, Department of Neurosurgery, Istanbul, Turkey \\ ${ }^{4}$ Ministry of Health, Silivri State Hospital, Department of Neurosurgery, Istanbul, Turkey \\ ${ }^{5}$ University of Health Sciences, Bakirkoy Sadi Konuk Training and Research Hospital, Department of Neurosurgery, Istanbul, Turkey \\ ${ }^{6}$ Turkish Republic Ministry of Justice, Forensic Medicine Institution, Istanbul, Turkey
}

Corresponding author: Baris PEKER barispekermd@gmail.com

\section{ABSTRACT}

AIM: To examine the morphological structure of anterior lumbar spinal region.

MATERIAL and METHODS: Fifteen fresh human cadavers were studied in our forensic medicine institution. Within the first 24 hours after death, cadavers were subject to a routine autopsy, by which the intestines were removed and the structures adjacent to the lumbar arteries (LAs) were examined. The following characteristics of the LA were examined and recorded: diameter, number of LA, intervertebral disc, and vertebral corpus relationship, and LA anatomical variations.

RESULTS: In 14 of the 15 cadavers (93.3\%), the first four LAs originated from the abdominal aorta. In one (6.7\%) cadaver, the fourth LA originated from the right aortic trunk together with the median sacral artery (MSA), and this was observed only in the right side. In one cadaver, the first LAs emerged as a common trunk. Twelve cadavers did not have a fifth LA. In one cadaver (6.7\%), the fifth LAs emerged as in a pair, distally from the origin of the MSA. Two cadavers (13.3\%) had fifth LAs that emerged from the common trunk with the MSA and this occurred only on the right side. LAs which emerged from the right side of the abdominal aorta passed under the inferior vena cava. Of the first four LA, the third LA had the largest vessel diameter, and the first LA had the narrowest vessel diameter. Fifth LAs had one of the narrowest vessel diameters of all LAs.

CONCLUSION: We consider that anatomical study of LAs using fresh cadavers can be useful to ensure safer anterior lumbar spinal surgeries.

KEYWORDS: Anterior lumbar spinal surgery, Cadaver, Lumbar arteries, Forensic medicine

\section{INTRODUCTION}

$\mathrm{T}$ lechnical advances in anterior lumbar spinal surgery have led to an increase in surgical interventions for infections, congenital and degenerative deformities, traumatic fractures, and primary and secondary neoplastic lesions (14). With an anterior lumbar approach, injury to the lumbar arteries (LAs) is frequently encountered (17). LAs originate from the posterolateral side of the abdominal aorta and are usually four pairs of arteries $(3,5)$. LAs that correspond to
Halil CAN (1) : 0000-0002-5699-4089

Furkan DIREN (1) : 0000-0001-6169-9722

Baris PEKER (1) : 0000-0001-8146-5210
Cengiz GOMLEKSIZ

(1) : 0000-0003-4789-5633

Dogan Gucluhan GUCLU (1) : 0000-0002-9959-961X

Ceyhun KUCUK (10): 0000-0001-5372-3753
Erdinc CIVELEK (10): 0000-0002-3988-4064

Aydin AYDOSELI (1) : 0000-0002-4695-8295

Altay SENCER (1) : 0000-0001-9925-5422 
the L1, L2, L3, and L4 vertebral bodies are called first, second, third, and fourth LAs, respectively (8). The median sacral artery (MSA) is a small branch which usually emerges above the aortic bifurcation, from the posterior side (3). Lower LAs generally originate from a common trunk near the midline of the posterior aorta. These arteries proceed laterally along the lumbar vertebral bodies. LAs are divided into anterior and posterior branches at the medial border of the psoas muscle and the anterior border of the transverse process (5). Although generally the four pairs of LAs originate from the posterior side of the aorta, these can arise from the MSA in a fifth pair (15).

LAs are the somatic segmental branches of the abdominal aorta, which correspond to the intercostal branches of the thoracic aorta. Although the LAs provide the blood supply to muscles, joints, ligaments, fascia, vertebral column, and intervertebral discs, these are also known to provide arterial support to the spinal cord (20). In rare cases of surgeries with an anterior lumbar approach, the ligation of the LAs has resulted in a severe neurological deficit. Ligation of the LAs during surgical intervention intended to repair thoracoabdominal aortic aneurysms reportedly resulted in paraplegia (20). Additionally, it is known that atherosclerotic obliteration of LAs and MSA contributes to the degeneration of intervertebral discs (9). Therefore, the anatomy of the LAs should be well known before embarking on anterior lumbar spinal surgery.

In cadaver dissections and postmortem arteriographic studies, arterial feeding of the lumbar spine has been described to supply by means of four pairs LA originating from the posterior of the aorta and in some cases by the fifth pair LAs originating from the MSA (15). Many studies have reported variations in retroperitoneal lumbar vessels. Thus, the aim of our study was to investigate the relationship between the LA and adjacent anatomical structures and to determine anatomical variations as we consider that this knowledge will contribute to a decrease in complications of anterior lumbar approaches. We performed our study using fresh cadavers as we consider this source of data will provide a better and more accurate understanding of the surgical anatomy.

\section{MATERIAL and METHODS}

\section{Study Design}

The study was conducted in 2015 at the Morgue Department of Istanbul Forensic Medicine Institute, after receiving approval of the ethics committee (No: 21589509/387; Date: 21.04.2015). The sample comprised 15 fresh human cadavers. Of the 15 cadavers included in the study, 8 were male and 7 were female and their age ranged between 27 and 58 years (mean age, 40.8 years). The cadavers included in the study were selected from the bodies that had not undergone abdominal surgery and had not suffered intra-abdominal or spinal injuries.

\section{Procedures}

The cadavers were placed in the supine position on the autopsy table. A routine median autopsy incision was performed followed by ligation and removal of the intestines. The abdom- inal cavity of each body was then examined to identify the location at which the LAs emerged from the abdominal aorta along with any anatomical variants of the LAs, the diameter of each LA, the relationship between the MSA and LAs, and the relationship of LAs with intervertebral discs and inferior vena cava. The levels at which the LAs emerged from the abdominal aorta were defined in terms of their relationship with the intervertebral disc as upper, middle and lower $1 / 3$ of the vertebral corpus. For measurements of LA diameters, the LAs were digitally photographed with a marker and were then digitally evaluated using ImageJ software (NIH, Bethesda, MD, USA). Descriptive statistical analysis was used to determine the mean, standard deviation (SD), and minimum and maximum values.

\section{Statistical Analysis}

SPSS Statistics version 22.0 (IBM, Armonk, NY, USA) was used for statistical analysis while evaluating the findings of the study. While evaluating the study data, the suitability of the parameters in terms of normal distribution was evaluated with the Shapiro Wilks test. Accordingly, parameters were found to be suitable for a normal distribution. In addition to describing results using descriptive statistics (i.e., mean, SD, minimum, maximum, and frequency), comparisons of quantitative data and between LA groups (parameters exhibiting a normal distribution) were performed using a one-way ANOVA test. To assess resulting in differences groups, the Tukey HDS test was used. A paired sample t-test was used for right and left side comparisons. Significance was evaluated at $\mathrm{p}<0.05$.

\section{RESULTS}

We examined 15 cadavers and found that the first four LAs emerged as pairs in 14 cadavers (93.3\%). $1 / 15$ cadaver $(6.7 \%)$ had a single right fourth LA originating from the common trunk along with the MSA (Figure 1). The first, second, and third LAs arose as pairs in all cadavers. In 1/15 cadaver, the first LA pair arose from abdominal aorta with the common trunk. Fifth LAs were found in $3 / 15$ cadavers. In $1 / 15$ cadaver $(6.7 \%)$ had a double fifth LA which originated distal MSA as left and right fifth LA. In 2/15 cadavers (13.3\%) had single fifth LA on the right side which was crossing the intervertebral disc and emerged from the common trunk with the MSA (Figure 2). LAs on the right side of the abdominal aorta passed under the vena cava (Figure 3). The level at which the right and left LAs originated from the abdominal aorta relative to the vertebral corpus were same level at both side (Figure 4).

Levels of first four pairs of LAs emerged from the abdominal aorta ( $n=118$ LAs) were evaluated according to the vertebral corpus; $49.1 \%$ (58 LA) from the middle $1 / 3$ of the vertebral corpus, $35.6 \%$ (42 LA) from the lower 1/3, and 15.3\% (18 LA) from the upper $1 / 3$ were seen. In $1 / 15$ cadavers $(6.7 \%)$, the fourth LA that came out of common trunk with MSA passed the intervertebral disc. The first and second LAs originated mostly from the middle and lower $1 / 3$ of the vertebral corpus, while the third and fourth LAs originated mostly from the upper and middle $1 / 3$ of the vertebral corpus. Exit levels of the first four lumbar artery pairs according to the vertebral bodies in Table I. All the fifth LAs, seen in three cadavers, were crossing the intervertebral disc (Figure 5). 
Table I: Exit Levels of the First Four Lumbar Artery (LA) Pairs According to the Vertebral Bodies

\begin{tabular}{lccccc}
\hline \multirow{2}{*}{ Side } & LA-vertebral body & First LA & Second LA & Third LA & Fourth LA \\
\cline { 3 - 6 } & lower part 1/3 & $\mathbf{n ~ ( \% )}$ & $\mathbf{n}(\%)$ & $\mathbf{n}(\%)$ & $\mathbf{n}(\%)$ \\
\hline Right & middle part 1/3 & $7(46.7)$ & $8(53.3)$ & $4(26.7)$ & $2(14.3)$ \\
\hline & upper part 1/3 & $1(6.7)$ & $7(46.7)$ & $10(66.7)$ & $5(35.7)$ \\
\hline Left & lower part 1/3 & $7(46.7)$ & $8(53.3)$ & $1(6.7)$ & $7(50)$ \\
\hline & middle part 1/3 & $7(46.7)$ & $7(46.7)$ & $10(66.7)$ & $5(35.7)$ \\
\hline \multirow{2}{*}{ Total } & upper part 1/3 & $1(6.7)$ & $0(0)$ & $1(6.7)$ & $7(50)$ \\
\hline & lower part 1/3 & $14(46.7)$ & $16(53.3)$ & $8(26.7)$ & $4(14.3)$ \\
\hline & middle part 1/3 & $14(46.7)$ & $14(46.7)$ & $20(66.7)$ & $10(35.7)$ \\
\hline
\end{tabular}

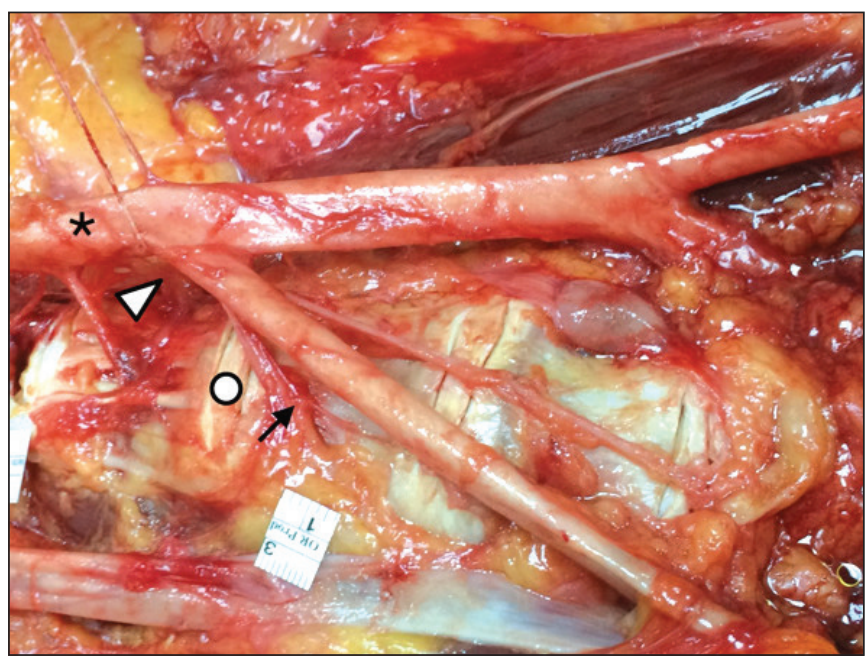

Figure 1: Fourth LA originating from the common trunk along with the MSA. Arrow: fourth LA, arrow head: MSA and fourth common trunk, asteriks: aorta, circle: intervertebral disc.

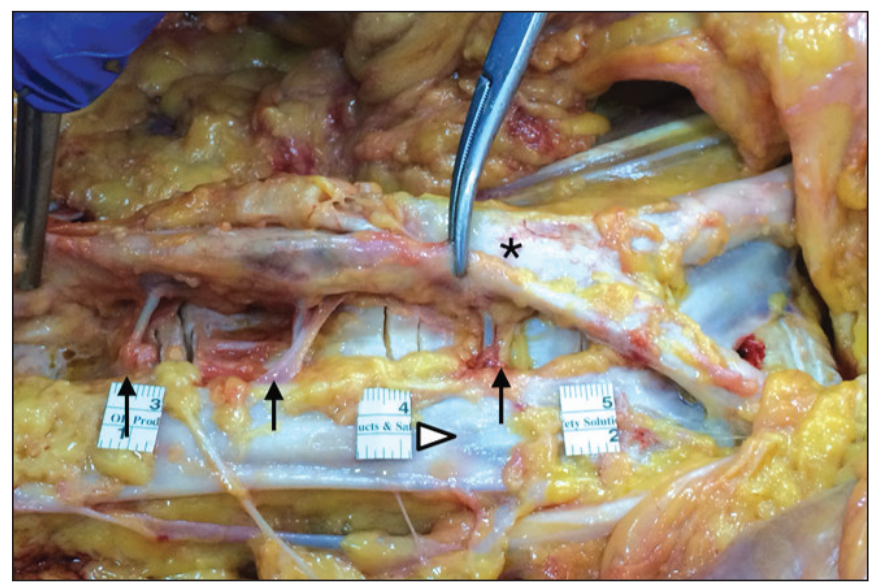

Figure 3: Relationship of the lumbar arteries originating from the abdominal aorta with the inferior vena cava. Arrow: LAs, arrow head: vena cava inferior, asteriks: aorta.

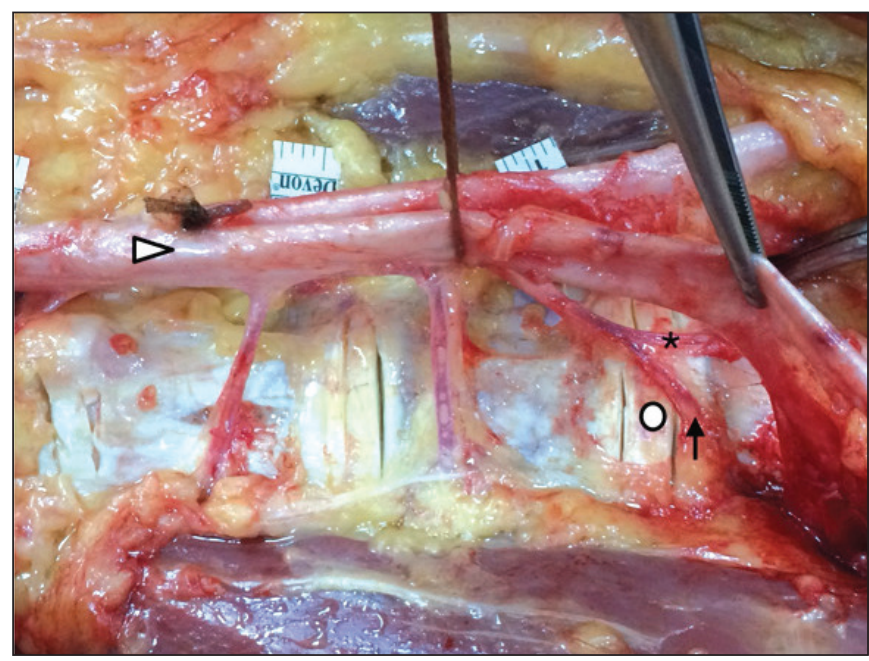

Figure 2: Fifth LA crossing the intervertebral disc and arised from the common trunk with the MSA. Arrow: fifth LA, arrow head: aorta, asteriks: MSA, circle: intervertebral disc.

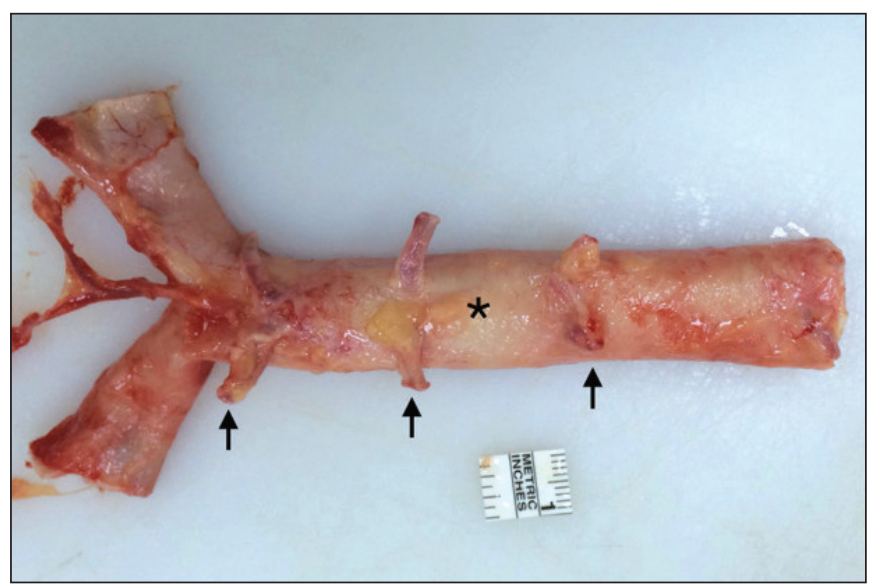

Figure 4: The posterior surface of the abdominal aorta, which was resected with the iliac arteries and LAs. Arrow: LAs, asteriks: aorta. 


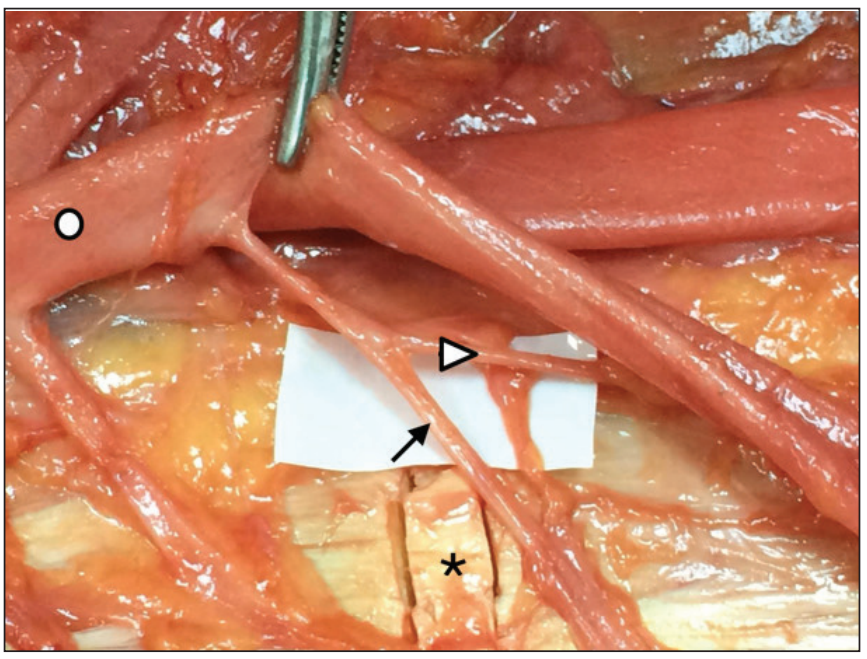

Figure 5: Image of the cadaver where the fifth lumbar artery crosses the intervertebral disc. Arrow: fifth LA, arrow head: median sacral artery, asterics: intervertebral disc, circle: aorta.

Third LAs had the largest vessel diameter among the first four LAs, and the mean vessel diameter was $2.28 \pm 0.36 \mathrm{~mm}$. First LAs had the narrowest vessel diameter among the first four LAs, and the mean vessel diameter was $1.67 \pm 0.38 \mathrm{~mm}$. The mean vessel diameter of the fifth LA, which were found in three cadavers, was measured $1.48 \pm 0.71 \mathrm{~mm}$ (Table II).

There was no statistically significant difference between the mean widths of the right and left first, second, third, and fourth LA pairs $(p>0.05)$. There was a statistically significant difference between the mean diameter of the first, second, and third LAs and fourth LA ( $p=0.000 ; p<0.05)$.

Statistically significant differences by the level at which the LAs originated was analyzed by binary comparisons; the mean diameters of the third LAs $(p=0.006)$ were found to be statistically significantly higher than the first $(p=0.000)$, second $(p=0.000)$ and fourth LAs $(p<0.05)$. The mean diameter of the fourth LA was found to be statistically significantly higher than that of the first LA $(p=0.027 ; p<0.05)$ (Table III).

\section{DISCUSSION}

Ito et al. first described the anterior lumbar approach for lumbar spine surgery in 1934 to treat Pott's disease (7). Since then, this approach has been the mainstay of spine surgery. Surgical procedures, including anterior surgical decompression and fusion, are increasingly being used for the treatment of various pathological processes of the spine, including trauma, deformity, infection, degenerative diseases, metastasis, and primary spinal neoplasms $(14,17)$. Advances in spinal fusion techniques and artificial disc replacement have led to an increase in the need for anterior retroperitoneal exposure of the lumbar spine. With such anterior retroperitoneal exposure of the lumbar spine, the risk of vascular injury increases. Moreover, such injuries require further operative repair (6). Nevertheless, the manipulation of the LA may still be necessary while performing anterior retroperitoneal approaches.
Table II: Diameters of Lumbar Arteries (LAs)

\begin{tabular}{|c|c|c|c|c|}
\hline \multirow[b]{2}{*}{ LA } & \multirow[b]{2}{*}{ Side } & \multirow[b]{2}{*}{$\mathbf{n}$} & \multicolumn{2}{|c|}{ Width (mm) } \\
\hline & & & Mean \pm SD & Range \\
\hline \multirow[t]{3}{*}{ First LA } & Right & 15 & $1.62 \pm 0.33$ & $1.09-2.16$ \\
\hline & Left & 15 & $1.71 \pm 0.44$ & $1.23-2.93$ \\
\hline & All & 30 & $1.67 \pm 0.38$ & $1.09-2.93$ \\
\hline \multirow[t]{3}{*}{ Second LA } & Right & 15 & $1.8 \pm 0.38$ & $1.12-2.37$ \\
\hline & Left & 15 & $1.8 \pm 0.39$ & $1.19-2.38$ \\
\hline & All & 30 & $1.8 \pm 0.38$ & $1.12-2.38$ \\
\hline \multirow[t]{3}{*}{ Third LA } & Right & 15 & $2.27 \pm 0.36$ & $1.73-2.85$ \\
\hline & Left & 15 & $2.29 \pm 0.38$ & $1.79-3.13$ \\
\hline & All & 30 & $2.28 \pm 0.36$ & $1.73-3.13$ \\
\hline \multirow[t]{3}{*}{ Fourth LA } & Right & 15 & $1.94 \pm 0.42$ & $1.35-2.66$ \\
\hline & Left & 14 & $1.95 \pm 0.37$ & $1.49-2.74$ \\
\hline & All & 29 & $1.95 \pm 0.39$ & $1.35-2.74$ \\
\hline \multirow[t]{3}{*}{ Fifth LA } & Right & 3 & $1.57 \pm 0.84$ & $0.98-2.53$ \\
\hline & Left & 1 & 1.18 & \\
\hline & All & 4 & $1.48 \pm 0.71$ & $0.98-2.53$ \\
\hline
\end{tabular}

Table III: Evaluation of Diameters Between Lumbar Artery (LA) Groups

\begin{tabular}{lccc}
\hline & \multicolumn{3}{c}{ Width $(\mathbf{m m})$} \\
\hline LA & Mean \pm SD & Mean \pm SD & Mean \pm SD \\
\hline First LA & $1.62 \pm 0.33$ & $1.71 \pm 0.44$ & $1.67 \pm 0.38$ \\
\hline Second LA & $1.80 \pm 0.38$ & $1.80 \pm 0.39$ & $1.80 \pm 0.38$ \\
\hline Third LA & $2.27 \pm 0.36$ & $2.29 \pm 0.38$ & $2.28 \pm 0.36$ \\
\hline Fourth LA & $1.94 \pm 0.42$ & $1.95 \pm 0.37$ & $1.95 \pm 0.39$ \\
\hline $\mathbf{p}$ & $\mathbf{0 . 0 0 0 *}$ & $\mathbf{0 . 0 0 1 *}$ & $\mathbf{0 . 0 0 0 *}$ \\
\hline
\end{tabular}

One-way ANOVA Test * $p<0.05$

LA: lumbar artery, SD: standard deviation.

The anterior retroperitoneal approach to the lumbar region requires considerable surgical experience, a thorough knowledge of this anatomical region, and knowledge of possible variations of the most important vascular structures in the retroperitoneal region. Thus, knowing the anatomy and possible anatomical variants of the LAs will contribute to a decrease in the probability of LA injury which can lead to catastrophic consequences.

During anterior instrumentation of lumbar vertebra, several LAs may need to be ligated. Although the incidence of ischemic myelopathy after segmental vessel ligation is very low, this 
is a very serious complication $(11,12,19)$. During the anterior lumbar approach, retraction beneath the aorta may cause accidental ruptures of LAs and may result in bleeding that is difficult to control (17). Also LAs are particularly vulnerable to retraction injury during mobilization of the psoas major if done without sufficient care. Bleeding and complications can be prevented if the radicular vessels are mobilized appropriately. Traditionally, the authors have preferred a left-sided approach because of the technical simplicity of aortic repair (1).

Antoine Portal, using a simple dissection technique in 1803, stated that the LAs originated from the aorta in four pairs and that the fifth pairs originated from MSA (13). Ratcliffe in his study of the arterial anatomy of 93 vertebrae in 24 adult cadavers in 1980, found four pairs of LAs and stated that each LA is divided into various branches in the intervertebral foramina. He reported that the fifth LA pair emerged from the MSA and these arteries were smaller than the other LAs. He noted that there were anastomoses at all levels among LAs, but the most prominent was among the fourth and fifth LAs (16).

Baniel et al. performed a retroperitoneal dissection of 102 human cadavers. They observed individual variations in the total number of LAs originating from the aorta rather than the course of LAs and reported that LAs followed a regular pattern. They stated that the LA number was between two and four pairs and that $60 \%$ had three pairs (2). Songur et al. stated in their cadaver study that there were three pairs of LAs in $11.5 \%$ of the cases, and in 3 cases $(3.1 \%)$ the third and fourth LA originated from a common trunk (18).

In Ratcliffe's study on 1982, the fourth LA was observed in 63 of 100 cases. He reported that in 15 cases, the fourth LA originated from the short, single, midline artery on the posterior surface of the aorta, and in three cases, the third LA pair originated from the single, posterior midline artery. In two cases, the fourth LA originated from the MSA under a high bifurcation of the aorta. Fifth LAs were reported to be completely or partially imaged on both sides in $38 \%$ of cases. Fifth LAs were unilateral in 14 cases (15).

Karunanayake and Pathmeswaran studied 109 cadavers and reported that some pairs of LAs originate from a common stem from the abdominal aorta. They reported that the majority of cadavers (84\%) had four pairs of LAs and MSA. They reported that the first LA pair was found in $99 \%$ of cadavers, but in one cadaver, the first LA originated from a common stem. The second LA pair is present in $99 \%$ of cadavers and arose from a common root in $2 \%$ of cadavers. The third LA pair was found in $98 \%$ of cadavers and originated from a common root in $3 \%$ of the cadavers. The fourth LA pair was present in $83 \%$ of cadavers and $11 \%$ of the cadavers originated from a common root. They reported that the fifth LA pair was present in only one cadaver, in which the fifth LA pair and MSA originated from a common root in the left iliac artery. They stated that in one cadaver, the fourth LA arose from a common root with the MSA and in another cadaver the fifth $L A$ arose from a common root with the MSA (8).
Caglar et al. reported that the diameter of the right LA was generally larger than that of the left LA in their study of 16 cadavers. They reported that the fifth LA originated from the MSA or iliolumbar artery (4). In contrast with the findings reported by Ratcliffe, they reported that the fifth LA diameter was the largest (15).

Arslan et al. examined 80 LAs in 15 cadavers and reported that four pairs of LAs originated from the dorsal aspect of the aorta in all specimens. In one of the specimens, they showed that the right and left LA originated from a common trunk at both L3 and L4 levels. They reported that the fourth LA had the largest diameter and the first LA had the smallest diameter (3.25 $\mathrm{mm}$ and $2.31 \mathrm{~mm}$, respectively). They reported that LA diameters increased slightly from L1 to L4 (1). Kirici et al. stated that there was no significant difference between the diameters of the right and left LAs in their study of 15 cadavers (10).

The numbers of the first four pairs of LAs in our fresh cadaver study were similar to those reported by Arslan et al. and Karunanayake and Pathmeswaran, but the findings of Baniel et al. differed from ours $(1,2,8)$. Only one cadaver $(6.7 \%)$ had a right fourth $L A$ originating from the common trunk with MSA instead of the fourth LA pair. The first, second and third LAs arose as pairs in all cadavers. In one cadaver, the first LA pair arose from the common trunk. Fifth LAs were not found in twelve cadavers. Only one cadaver $(6.7 \%)$ had a pair of fifth LAs and originated distally from the MSA as the left and right pairs. Two cadavers (13.3\%) had a single fifth LA on the right that originated from the common trunk with MSA.

Caglar et al. reported that the fifth LA diameter was the largest (4). Arslan et al. reported that the fourth LA had the largest diameter and the first LA had the smallest diameter $(3.25 \mathrm{~mm}$ and $2.31 \mathrm{~mm}$, respectively) (1). In contrast with those previous reports, in our study, the widest vessel diameter from the first four lumbar arteries was in the third lumbar arteries and mean vessel diameters were measured as $2.28 \pm 0.36 \mathrm{~mm}$. The first LA had the narrowest vessel diameter with a mean vessel diameter of $1.67 \pm 0.38 \mathrm{~mm}$, while the fifth LA had the smallest vessel diameter of all LAs, with a mean vessel diameter of $1.48 \pm 0.71 \mathrm{~mm}$ (Table II).

The third and fourth LAs arose mostly from the upper and middle $1 / 3$ of the vertebral bodies while the first and second LAs arose mostly from the lower and middle $1 / 3$ of the vertebral body (Table I). In our study, fifth LAs were only detected in three cadavers, and these crossed through the intervertebral disc. As it can be understood from the results of our study, since the first three pairs of lumbar arteries are located further away from the intervertebral disc distance, they are less at risk for injury during the anterior retroperitoneal approach. However due to very few of the fourth lumbar arteries cross the intervertebral disc distance, the risk is slightly higher than others. Since the fifth lumbar arteries keep going by crossing the L5-S1 disc levels, the risk of lumbar artery damage is considerably higher than other disc levels. We consider that the data collected in our study of 15 cadavers, consisting of measurements very close to the actual surgical anatomical values, will build on the existing literature. Additionally, these data may contribute to 
increase the success rates and reduce the complication rates of anterior retroperitoneal lumbar surgery.

\section{CONCLUSION}

Anterior retroperitoneal surgery has become a widely used approach for spine pathologies. Bleeding from LAs during anterior retroperitoneal surgery for the lumbar spine is a potentially relevant complication because this situation may cause serious consequences. Understanding the possibility of LA variations and knowing the relationship between the output levels of LAs and the intervertebral disc are helpful for spine surgeons embarking on anterior spine surgery as this information may help reduce the risk of procedural complications to a minimum. Surgeons need to be more careful during retroperitoneal exposure to avoid complications. This may be possible by a greater understanding of the anatomy of the anterior retroperitoneal region.

\section{ACKNOWLEDGMENTS}

The addresses for the author with asterisk(s) are as follows:

*Medicine Hospital, Neurosurgery Clinic, Istanbul, Turkey

\section{REFERENCES}

1. Arslan M, Comert A, Acar HI, Ozdemir M, Elhan A, Tekdemir I, Tubbs RS, Ugur HS: Surgical view of the lumbar arteries and their branches: An anatomical study. Neurosurgery 68 Suppl Operative 1:16-22, 2011

2. Baniel J, Foster RS, Donohue JP: Surgical anatomy of the lumbar vessels: Implications for retroperitoneal surgery. J Urol 153(5):1422-1425, 1995

3. Borley NR: Posterior abdominal wall and retroperitoneum. In: Gray's Anatomy. 39th ed. Edinburgh: Churchill Livingstone, 2005: 1116-1119

4. Caglar S, Dolgun H, Ugur HC, Torun F, Tekdemir I, Elhan A: Extraforaminal lumbar arterial anatomy. Surg Neurol 61(1):2933, 2004

5. Chan KT, Korivi N: Lumbar artery pseudoaneurysm in traumatic spinal cord injury: A case report. Arch Phys Med Rehabil 84:455-457, 2003

6. Garg J, Woo K, Hirsch J, Bruffey JD, Dilley RB: Vascular complications of exposure for anterior lumbar interbody fusion. J Vasc Surg 51(4):946-950, 2010
7. Ito H, Tsuchiya J, Asami G: A new radical operation for Pott's disease. J Bone Joint Surg 16:499-515, 1934

8. Karunanayake AL, Pathmeswaran A: Anatomical variations of lumbar arteries and their clinical implications: A cadaveric study. ISRN Anat 2013:154625, 2013

9. Kauppila LI: Can low-back pain be due to lumbar-artery disease. Lancet 346(8979):888,889, 1995

10. Kirici Y, Yazar F, Yalcin B, Kocabiyik N, Sanisoglu Y, Ozan $\mathrm{H}$ : Morphology of the lumbar arteries. Gülhane Tıp Dergisi 46(4):323-328, 2004

11. Leung YL, Grevitt M, Henderson L, Smith J: Cord monitoring changes and segmental vessel ligation in the "at risk" cord during anterior spinal deformity surgery. Spine (Phila Pa 1976) 30(16):1870-1874, 2005

12. Orchowski J, Bridwell KH, Lenke LG: Neurological deficit from a purely vascular etiology after unilateral vessel ligation during anterior thoracolumbar fusion of the spine. Spine (Phila Pa 1976) 30(4):406-410, 2005

13. Portal PA: Cours d'Anatomie Medicale ou Elementes de l'Anatomie de l'Homme. Paris: Baudouin, 1803:295-296

14. Rajamaran V, Vingan R, Roth P, Heary RF, Conklin L, Jacobs GB: Visceral and vascular complications resulting from anterior lumbar interbody fusion. J Neurosurg 91 Suppl 1:6064, 1999

15. Ratcliffe JF: The anatomy of the fourth and fifth lumbar arteries in humans: An arteriographic study in one hundred live subjects. J Anat 135(Pt 4):753-761, 1982

16. Ratcliffe JF: The arterial anatomy of the adult human lumbar vertebral body: A microarteriographic study. J Anat 131(pt 1):57-79, 1980

17. Samudrala S, Khoo LT, Rhim SC, Fesler RG: Complications during anterior surgery of the lumbar spine: An anatomically based study and review. Neurosurg Focus 7(6):9, 1999

18. Songur A, Toktas M, Alkoc O, Acar T, Uzun I, Bas O, Ozen OA: Abdominal aorta and its branches: Morphometry - variations in autopsy cases. Eur J Gen Med 7(3):321-325, 2010

20. Tsirikos Al, Howitt SP, McMaster MJU: Segmental vessel ligation in patientsundergoing surgery for anterior spinal deformity. J Bone Joint Surg Br 90(4):474-479, 2008

19. Williams PL, Bannister LH, Berry MM, Collins P, Dyson M, Dussek JE, Ferguson MW: Gray's anatomy: The anatomical basis of medicine and surgery. $38^{\text {th }}$ ed, New York: ChurchillLivingstone, 1995:1558 